\title{
Finite Element Simulation for Dynamic Performance of a Dummy's Head in Helicopter Anti-prang Tests
}

\author{
Jingfa Lei ${ }^{1,2}$, Yan Xuan ${ }^{2}$, Tao Liu ${ }^{2, a}$, Miao Zhang ${ }^{2}$ and Hong Sun ${ }^{2}$ \\ ${ }^{1}$ State Key Laboratory of Vehicle NVH and Safety Technology, Chongqing 401120, China \\ ${ }^{2}$ School of Mechanical and Electrical Engineering, Anhui Jianzhu University, Hefei 230601, China
}

\begin{abstract}
The dynamic performance of a dummy's head plays a important role in the research of helicopter anti-prang testing. In this paper, we firstly construct the finite element model for a dummy's head based on the parameter features of 50th percentile Chinese pilot. Then, the dynamic performance of the head model is simulated according to the calibration rules. After comparing the simulation results with the reference index of the dummy head's dynamic performances, we find that the FE model has good anthropopathic integrality, and the simulation method used to analyze the dynamic performance of the dummy's head is correct and validated. This paper has practical guiding significance in the study of helicopter anti-prang testing and other dummy parts, which also provide the reference value for the improvement of dummy structure.
\end{abstract}

\section{Introduction}

A dummy in helicopter anti-prang tests is an important tool to test the crashworthiness and the protective ability of helicopters ${ }^{[1]}$. The dummy must have similar dynamic performance with the human body in order to reflect the dynamic response of the human body effectively under the same test conditions. The pilot's head is very prone to be seriously injured in a helicopter crash accident. Statistically, the head injury is roughly $25 \%$ of the total injuries in helicopter accidents, whose death rate is about $31.5 \%{ }^{[2]}$. Therefore, the dynamic performance of the head model directly determines the quality of the developed dummy in helicopter anti-prang tests.

The dynamic performance of a dummy's head can be analyzed by experimental test and computer simulation. In the experimental test, a certain load is applied on the dummy's head according to the main injuries of human head in helicopter accidents and corresponding regulations. The impact response of the dummy's head is detected by the sensor installed on the head model's mass center, and the parameters such as acceleration are measured, collected and analyzed dynamically. While in computer simulation, the finite element model of the dummy's head is established, the head's calibration test process is simulated in simulation software, and then the dynamic response parameters of the dummy's head are obtained which can be used to evaluate whether the head model meets the crash test requirements according to corresponding regulations. The key in computer simulation is to construct the simulation model of the dummy's head, and multi- rigid body model and finite element model are the main computer simulation models, among which the finite element model is considered to be the most effective model when analyzing the collision problems of high-speed vehicles. Some experience has been accumulated in the construction and application of the dummy's finite element model. The companies such as American Humanetics, LSTC, Holland TNO and German DYNAmore have established Hybrid III dummy's finite element model which have been widely used in academic and industrial industry. A dummy's finite element model has also been built in many simulation softwares such as LS-DYNA and PAM-SAFE. And some institutions and scholars have discussed and studied the finite element model of a dummy's head ${ }^{[3-5]}$. Although the dynamic performance of a dummy's head has been studied extensively at home and abroad, the researchers are mainly focused on the vehicle frontal and side crash test dummy, and more on foreign dummies. The collision and damage forms of an anti-prang dummy's head in tests are more complicated relatively, and few studies have been carried on the dynamic response characteristics of its head model in the complicated state. The dummy which can evaluate collision damages from different directions has not yet been developed because of the limitation of the dummy's manufacturing technology. As in Europe and the United States, Hybrid III dummy is used in helicopter anti-prang tests in China. According to the third pilot anthropometric data ${ }^{[6]}$, there are large differences between $50^{\text {th }}$ percentile Chinese pilot and those of Hybrid III $50^{\text {th }}$ dummy in height, weight and sitting height. Therefore, it cannot reflect the actual injury situations of human body accurately under impact load by using foreign dummies for the safety test of Chinese pilots. According to the third pilot anthropometric parameter

a Corresponding author: tao.liu@ahjzu.edu.cn 
measurement results and dummy design experience at home and abroad, a head model with head feature parameters of 50th Chinese pilot has been developed by Human Engineering Laboratory of Sichuan University, and the dynamic performance of the dummy's head has been tested based on the foreign similar dummy calibration methods ${ }^{[7]}$. On the basis of the research above, the geometric model and finite element model of the pilot dummy's head were constructed respectively in SolidWorks and ANSYS Workbench here. According to the calibration test requirements, the dynamic performance of the dummy's head was simulated in LS-DYNA software. And the response parameters of the head's dynamic performance were obtained. The feasibility of the method and the efficiency of the head's finite element model were verified by comparing the simulation results to the experimental results.

\section{INJURY INDEX OF THE DUMMY'S HEAD}

In order to evaluate the head injury easier, a variety of head injury evaluation criteria have been put forward after many years of research such as WSTC (Wayne State Tolerance Curve) ${ }^{[8]}$, $\mathrm{HIC}^{[9]}$ (Head Injury Criterion), $\mathrm{SI}^{[10]}$ (Severity Index), GAMBIT ${ }^{[11]}$ (Generalized Acceleration Model for Brain Injury Threshold), CSDM [12] (Cumulative Strain Damage Measure) and HIP [13] (Head Impact Power). And among them, HIC is the most widely used one, which can be obtained by calculating the integral of the head' resultant acceleration. The calculation formula is as follows:

$$
\begin{gathered}
H I C=\left\{\left(t_{2}-t_{1}\right)\left[\frac{1}{t_{2}-t_{1}} \int_{t_{1}}^{t_{2}} a(t) d t\right]^{2.5}\right\}_{\max } \\
a(t)=\sqrt{a_{x}^{2}+a_{y}^{2}+a_{z}^{2}}
\end{gathered}
$$

In the formula, $a(t)$ is for the resultant impact acceleration of the head's centroid, which is expressed as multiples of gravity acceleration. $t_{1}$ is the random time during the process of accelerating. $t_{2}$ is the time that HIC reaches the maximum value relative to $t_{1}$. The maximum time interval of pulse action, namely $t_{2}-t_{1}$, is set to $36 \mathrm{~ms}$ for convenience. But the time interval is suggested to be reduced to $15 \mathrm{~ms}$ later because it influences the calculation of HIC greatly. HIC $=1000$ is considered to be the safety limit when evaluates the human head's injury in present laboratory conditions. The standard has been widely used in the safety regulations of all around the world.

HPC (Head Performance Criterion), which quantifies the collision extent of a dummy's head when impacted, is used to evaluate the dynamic performance of a dummy's head in European regulations ECE R94 and R95. The definition and computation of HPC are the same as those of HIC when the maximum time interval is $36 \mathrm{~ms}$. The Chinese CMVDR294 standard also specifies that the dynamic performance index HPC of a dummy's head can't exceed 1000.

The injury form of the pilot's head is very complicated in a helicopter crash accident because of the influences of the helicopter's crash angle and mode, transverse and longitudinal velocity, and crash position and so on. In general, there are mainly three injury forms of human head in a helicopter accident as follows: (1) In a helicopter crash accident, human head may collide with the cabin media because of inertia, or helicopter blades may deform and pierce the body, or the transmission system may break down, which all may generate an impact load on the head. And once the impact load on the skull exceeds its elastic limit, injuries such as skull fracture may appear. (2) The impact load produced in the process of helicopter crash may cause impact wave in human body, and once the strength of the impact exceeds the limitation of brain, some hurt may appear. (3) The deceleration or acceleration of human head changes suddenly because the helicopter decelerates sharply in a crash accident. The amplitude of the head's acceleration curve is very high, which may cause the brain tissue deformation or movement relative to the skull, and the injuries such as subdural hematoma, intraventricular hematoma may appear. The load that the pilot's head bears during the crash is often the resultant of the loads above.

The collision and injury form of the pilot's head is very complicated in a helicopter crash accident because of the diversity and unpredictability of the helicopter's crash environment. And there are no standards that can be used to appreciate the dynamic performance of a helicopter anti-prang dummy's head presently. Compared with the injuries in a vehicle collision accident, human body bears an additional impact load in the vertical direction in a helicopter crash accident. Through analysis, it's found that the injury of the pilot's head in a helicopter accident is lighter than that of the occupant's head in a vehicle collision accident except the extreme situation that human head is crushed. Based on the current research results of human head injury biomechanics and foreign experiences of evaluating the dynamic performance of similar dummies such as Hybrid III dummy, the acceleration response of the centroid of the dummy's head has been regarded as the measurement criteria of its dynamic performance here, which can improve the safety factor of the head model for helicopter anti-prang dummy. And the regulation about the dynamic performance parameters of the dummy's head has been ascertained according to HPC. Only the dummy's head meets the regulation can the head model developed simulate the injury situation of human head properly in the crash environment.

To guarantee the repeatability and stability of the data measured in the collision test by transducer which is installed in the dummy's head, researchers have carried on many simulate impulse tests by using corpse's head and dummy's head. Comparing the response data of head collision between various shape and rigid surface, it is found that the collision data of rigid surface is the only one with good repeatability and strong adaptability. The mechanical response of the dummy's head fall freely from the given height onto the rigid horizontal is similar to that of the corpse's head. The variation characteristic of the head centroid's acceleration is measured when the head model fall freely from certain height and the model's forehead should contact with the rigid plane, 
which can be used to evaluate the dynamic response characteristics of dummy's head for domestic helicopter anti-prang.

It can improve the reliability of the head model developed that evaluates the dynamic performance of an anti-prang dummy's head with the standard of appreciating that of Hybrid III dummy head.

The parameter index of the dynamic performance of an anti-prang dummy's head is as follows:

(1) The resultant acceleration range of the centroid of the dummy's head is from $225 \mathrm{~g}$ to $275 \mathrm{~g}$, and the transverse acceleration range is from $-15 \mathrm{~g}$ to $15 \mathrm{~g}$.

(2) The main pulse of the head centroid's acceleration curve should have a single peak.

As HIC, only the influences of translational acceleration and action time are involved in HPC. Both HIC and HPC cannot predict injury situation of human's head caused by rotational acceleration accurately, and the influence of colliding direction hasn't been taken into account. HPC is used only in direct contact situation, and the impact time interval in HPC is also limited. HPC is thought to be able to distinguish between contact response and non-contact impact response. If there is no contact, there will be no calculation. The evaluation and measurement of the dynamic performance of the anti-prang dummy's head is needed further research and discussion.

\section{THE TEST CRITERION FOR THE DYNAMIC PERFORMANCE OF DUMMY'S HEAD}

According to GB 20071-2006, the head model is suspended during the experiment as in Figure 1. The suspension height is $376 \mathrm{~mm}$, the distance between the lowest points of the dummy forehead and its nose is $12.7 \mathrm{~mm}$. The center symmetry plane of the head should be in the vertical state. The head fall from the given height through the release device, and once it is released, it should fall onto the rigid horizontal steel plate with flat surface immediately ${ }^{[14]}$.

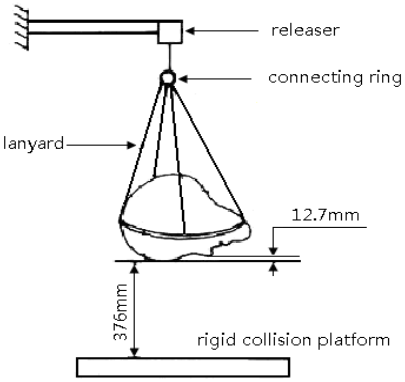

Figure 1. Test equipment sketch of the head's dynamic performance.

The height of the test device includes the release height $376 \mathrm{~mm}$, the head's height and the suspension height. The whole height shouldn't be less than $900 \mathrm{~mm}$. The collision platform should be kept horizontal, clean and dry, and the surface roughness value should be between $0.2 \mu \mathrm{m}$ and $2 \mu \mathrm{m}$. The weight of the cables of suspension lines and the accelerometer should be as light as possible in order to reduce the influence on the test results.

\section{CONSTRUCTION AND ANALYSIS OF DUMMY HEAD'S FINITE ELEMENT MODEL}

\subsection{Geometric model and material of the dummy's head}

The geometric model of the $50^{\text {th }}$ percentile pilot dummy's head has been established in SolidWorks according to the third pilot anthropometric parameter measurement results ${ }^{[5]}$. As shown in Figure 2, (a) is the front part of the skull, (b) is the back part, and (c) is the assembly of the head structure.

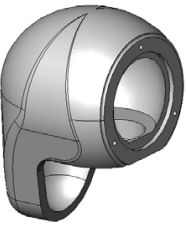

(a)

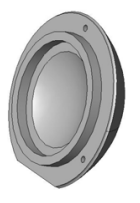

(b)

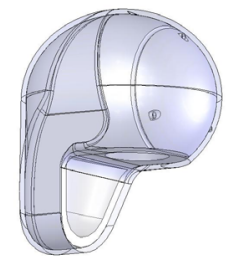

(c)
Figure 2. Head structure.

The skull is composed of two precision casting aluminum parts similar to human head's structure. The sensor is installed on an indicated position of the cavity. The skin that wraps the skull is firstly acted on by the impact load during collision. The silicone rubber composite material is developed independently by Human Engineering Laboratory of Sichuan University, and it is used to produce the dummy skin. The skin material can restore well after the deformation caused by positive force and resist shear force, and it is not easy to be torn. This material has a good biomechanical simulation. The density, Poisson ratio and elastic modulus of the composite material are $1038 \mathrm{~kg} / \mathrm{m}^{3}, 0.4$ and $1.3452 \mathrm{Mpa}$, respectively.

\subsection{Construction and analysis of the head's finite element model}

In order to reduce the difficulty of constructing the finite element model of a dummy's head and the scale of the model, and to improve the mesh generation quality and analysis speed, the geometric model of the head has been simplified. The key of studying the dynamic performance of a dummy's head is to obtain the change of the head centroid's acceleration when the head collides with the rigid surface. The facial features such as the eyes and nose as well as the structures such as chamfers and grooves have little influence on the analysis results, thus these factors will be omitted when constructing the head's geometrical model. The front and back part of the skull of the head model is actually tightly pressed together. These two parts will be merged into one part and the small grooves between them will be deleted when simplifying the model to make little influence on the mass and rigidity of the whole structure. The screw holes 
and threaded fasteners in the rear part can also be omitted.

The geometrical model of the dummy's head is constructed by SolidWorks software. The simplified head model is imported into ANSYS Workbench for the construction of the finite element model in order to ensure the data integrity of the head model and to prevent the failure of mesh generation. The finite element model of the dummy's head is obtained by element solid 164 and hybrid grid partition method, as shown in Figure 3. The finite element model is composed of 19537 elements and 23264 nodes.
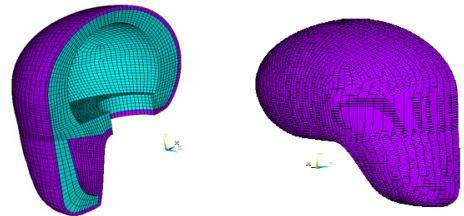

Figure 3. Meshing of the head model.

In order to improve computational efficiency, the element is integrated by reduced integration method. The calculation amount of the integration by the method is the least, but it may cause the structure unstable. So, it's necessary to control the hourglass mode. Energy analysis of the model is shown in Figure 4 and 5. The total internal energy of the model is 7.74J. The maximum value of the skin's hourglass energy is $0.11 \mathrm{~J}$, and the maximum value of the total hourglass energy is $0.368 \mathrm{~J}$, which is $4.75 \%$ of the total internal energy. Obviously, the percentage is lower than $10 \%$, which shows that the hourglass model is well controlled.

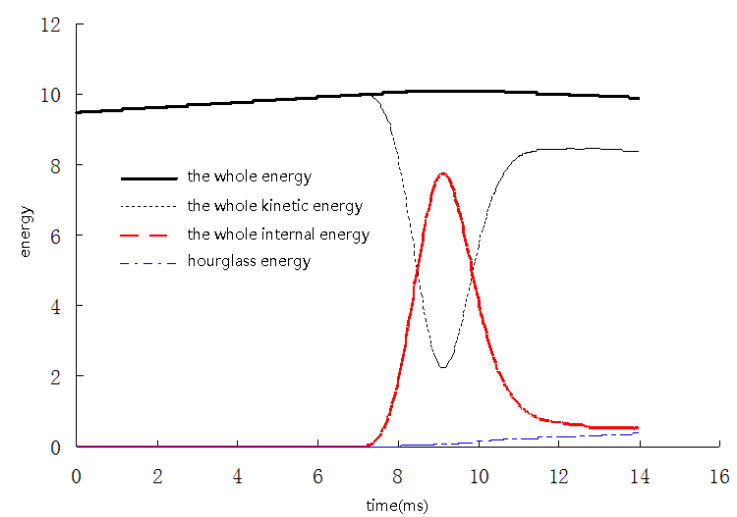

Figure 4. The whole energy change of the head's finite element model.

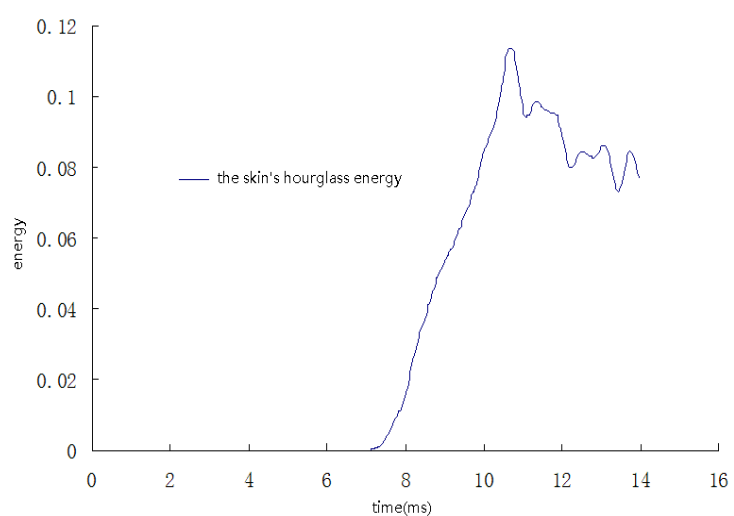

Figure 5. Hourglass energy change of the head's skin with time.

\section{SIMULATION OF THE HEAD'S DYNAMIC PERFORMANCE}

\subsection{Basic theories of the explicit dynamics}

In the analysis of dynamic response, there are many forms of time integration, among which the time step of the implicit algorithm method may be set bigger (such as 100-1000 times of that of the explicit algorithm method). But the stiffness matrix needs take inverse iteration in this method. If the system is large, the time step should be set smaller in order to obtain the response process, and then the amount of computer processing required is quite large. While in the explicit algorithm method, there's no need of establishing the stiffness matrix and the inverse operation. The algorithm is very simple, with little occupies memory, and can deal with nonlinear problems, and it has been widely used in the collision impact field.

Assuming that the current time step is $n$, the motion equation is:

$$
M \ddot{x}(t)+C \dot{x}(t)+K x(t)=F
$$

It can be expressed as:

$$
\begin{gathered}
M \ddot{x}\left(t_{n}\right)=F_{n}^{\text {ext }}-F_{n}^{\text {int }} \\
\ddot{x}\left(t_{n}\right)=M^{-1} F_{n}^{\text {residual }}
\end{gathered}
$$

Respectively, $\mathrm{n}$ is the $\mathrm{nth}$ time point; $F_{n}^{\text {ext }}$ is the external load; $F_{n}^{\text {int }}$ is the internal load; $F_{n}^{\mathrm{int}}=C \dot{x}\left(t_{n}\right)+K x\left(t_{n}\right) ; F_{n}^{\text {residual }}$ is the remaining force. The acceleration can be obtained by the transpose of the mass matrix and the remaining force.

$M$ is a diagonal matrix when the lumped mass matrix is used, and it's easy for the inversion. The linear equation group will become a series of independent linear equations of a degree about all the degrees of freedom, by which the acceleration can be calculated as

$$
\ddot{x}\left(t_{n i}\right)=F_{n i}^{\text {residual }} / M_{i}
$$

$M_{i}$ is for the mass of $i^{\text {th }}$ node. Time is cycled by the method of the central difference. $x(t)$ can be calculated at the time of $n+1 / 2$, namely:

$$
\dot{x}\left(t_{n+1 / 2}\right)=\dot{x}\left(t_{n-1 / 2}\right)+\frac{1}{2}\left(\Delta t_{n+1 / 2}+\Delta t_{n-1 / 2}\right) \ddot{x}\left(t_{n}\right)
$$

The new position is calculated at the time of $n+1$, namely:

$$
x\left(t_{n+1}\right)=x\left(t_{n}\right)+\dot{x}\left(t_{n+1 / 2}\right) \Delta t_{n+1 / 2}
$$

Due to the adoption of the lumped mass matrix, the solution of the above motion equations is uncoupled. It means that there is no relevance between the equations and it's unnecessary to form the overall matrix, which greatly save the storage space and computing time.

\subsection{Simulation of the dummy's head}

As shown in Figure 1, based on the explicit algorithm method, the dynamic performance of the dummy's head 
has been simulated by LS-DYNA according to the test requirements.

According to the boundary conditions and initial conditions, the head model would fall freely from the height of $376 \mathrm{~mm}$ and the model's forehead should contact with the rigid plane. In the simulation, the contact between the forehead and rigid collision surface is set to be point-surface contact and the acceleration of gravity applied on the head is $9.8 \mathrm{~m} / \mathrm{s}^{2}$. The displacement and speed of all the units of the model in direction $\mathrm{X}$ and $\mathrm{Z}$ should be zero and the collision platform should be a rigid body without degree of freedom. After repeating modification and debugging the model, the process during which the head model falls onto the rigid surface is simulated, and the acceleration curves in direction $\mathrm{X}, \mathrm{Y}$ and $\mathrm{Z}$ (after the average denoising) and the impact diagram of the head model are obtained respectively, as shown in Figure 6 and 7.

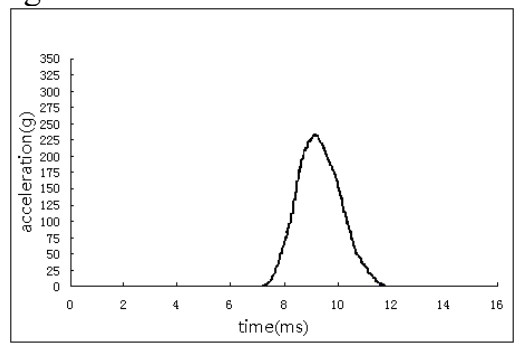

(a) Direction $\mathrm{X}$

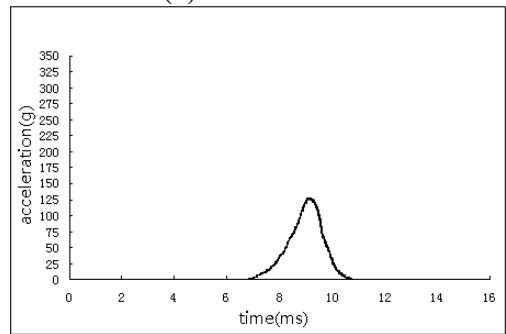

(b) Direction Y

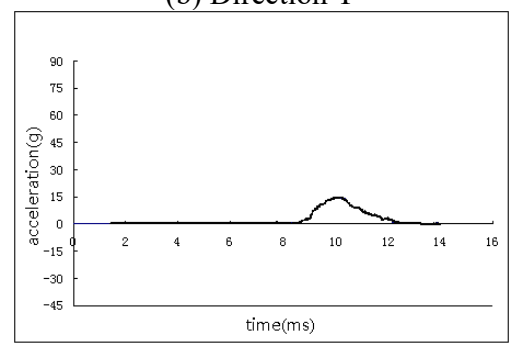

(c) Direction Z

Figure 6. Average acceleration curve of the dummy's head.

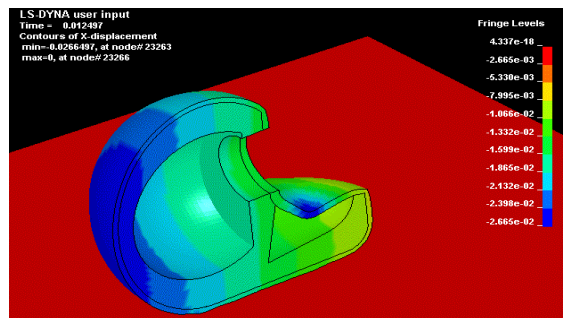

Figure 7. The impact result of the head.

As shown in Figure 6, all the acceleration curves of the head model in all direction have a single peak and the corresponding peak value of the three directions are $128.79 \mathrm{~g}(\mathrm{ax}), 233.12 \mathrm{~g}($ ay) and $14.97 \mathrm{~g}(\mathrm{az})$ respectively.
The maximal resultant acceleration is calculated by the equation (2), namely $a_{\max }=266.75 \mathrm{~g}$.

\section{ANALYSIS AND DISCUSSION OF THE RESULTS}

The main parameters of the dynamic performance of a dummy's head are the resultant acceleration and transverse acceleration of the head's centroid. Comparing the simulation results with the performance reference index of the head's dynamic performance, it's found that all the parameters of the head's dynamic performance are within the reference range, which shows that the dummy's head meets the requirements of collision tests. The value of the transverse acceleration is relatively large, which shows that the transverse wave is serious.

We make a comparison between the simulation results and experimental results ${ }^{[7]}$, as shown in Table 1.

Table 1. Comparison of the results obtained by test and

\begin{tabular}{|c|c|c|c|c|}
\hline Parameter & $a_{x}$ & $a_{y}$ & $a_{z}$ & $a_{\max }$ \\
\hline $\begin{array}{c}\text { Simulation } \\
\text { results }\end{array}$ & $128.79 \mathrm{~g}$ & $233.12 \mathrm{~g}$ & $14.97 \mathrm{~g}$ & $266.75 \mathrm{~g}$ \\
\hline Test results & $125.12 \mathrm{~g}$ & $203.3 \mathrm{~g}$ & $13.86 \mathrm{~g}$ & $239.22 \mathrm{~g}$ \\
\hline Error & $2.85 \%$ & $12.79 \%$ & $7.41 \%$ & $10.32 \%$ \\
\hline
\end{tabular}

As can be seen from Table 1, the error between the simulation results and test results is within allowable engineering range. The acceleration curves obtained by the two methods have the same waveform whose main pulse possesses a single peak shape.

The reason that causes the error between simulation results and test results may be as follows:

(1) In test, the inner sensor and cables are included in the tummy's head, but the mass of the sensor and the installation position hasn't been set in the simulation, which causes the difference of the results.

(2) The facial features of the dummy's head have been omitted when constructing its geometrical model, which will influence the inertial parameters of the model, thereby influencing the simulation results.

(3) The solid model of the dummy's head is made by hand, the machining accuracy is not high, and the materials used in the model may be inhomogeneous, which all may result in the difference of the mass, inertia parameters and symmetry between the solid model and the geometrical model.

(4) The position and mode of the head model's installation and release should accord with the requirements shown in figure 1 . But obviously, there are errors in the measurement of relevant distances and the location of the head model. The roughness and flatness of the rigid platform as well as the weight of the cable and accelerometer have influenced the test results. While in simulation, there are no such errors.

(5) It's found that the transverse acceleration both in simulation and test are high, which may be caused by the misalignment between the head model's horizontal axis and the reference coordinate. So the head model needs to be adjusted. 
(6) There might be differences between the material characteristic parameters of the simulation model and those of the solid model.

\section{CONCLUSIONS}

In this paper, the geometrical model and finite element model for a dummy's head with the $50^{\text {th }}$ percentile Chinese pilot parameter features have been constructed in SolidWorks and ANSYS Workbench respectively. And the dynamic performance of the head has been simulated by LS-DYNA according to its calibration rules. The simulation results are in accordance with the reference index of dynamic performance, which shows that the FE model has an excellent simulation performance. In addition, although there is an error between the experimental result and simulation result, it is acceptable considering the reasons analyzed above, which shows that the simulation method used to analyze the dynamic performance of the dummy's head is correct and validated. The finite element model for the dummy's head can be further used to analyze the dynamic performance of the dummy's neck.

The future work is as follows: constructing the FE model of helicopter anti-prang dummy with better details studying the dynamic performance of the dummy without tests; improving the structure, biological simulation, dynamic response characteristics, testing reproducibility, and sensitivity of the helicopter anti-prang dummy; exploring the test method and standard of a dummy's dynamic performance in complicated collision conditions constructing a series of dummies with the Chinese pilot's parameter features; laying a good foundation of computer simulation for the Chinese helicopter crash resistance tests.

\section{ACKNOWLEDGEMENTS}

This work is partially supported by Open Foundation of State Key Laboratory of Vehicle NVH and Safety Technology (NVHSKL-201407), Open Foundation of Key Laboratory of Testing Technology for Manufacturing Process (14zxzk01), the Nature Science Research Project of Education Department of Anhui Province (KJ2016A145, KJ2018A0519), the Natural Science Foundation of Anhui Province (1708085ME130), Key Project of the College Outstanding Young Talent Fund of Anhui Province (2013SQRL045ZD).

\section{REFERENCES}

1. H. M. Lankarani, M. G. Mirza. Parametric study of crashworthy bulkhead designs. DOT/FAA/AR-02/103. 2002.

2. Q. H. Zhu, Q. Sun. Advanced technologies for helicopter safety and survivability. Aviation Science and Technology. 5, (2005)

3. L. B. Cao, Y. P. Huang. Modeling and comparative study of finite element model for hybrid III 50th percentile dummy head. Automotive Engineering. 32, 2 (2010)

4. X. H. Lai, Z. Lin, Q. Zhou. Response surface-based optimization for validation of a finite element model of hybrid III head-neck sub-assembly. Altair 2010 HyperWorks

5. S. Chen, Z. F. Yuan, D. Q. Lin, etc. Design and Analysis of the Chinese crash dummy. Journal of Sichuan University (Engineering science edition). 40, 3 (2008)

6. Chinese people's Liberation Army General Armaments Department. The body size of the Chinese male pilots (GJB 4856-2003). National military standard of the People's Republic of China (2003)

7. Z. F. Yuan, D. Q. Lin, B. Zhou, etc. The production and calibration of the helicopter anti-prang experiment dummy[J]. Journal of Sichuan University (Engineering science edition). 39, 2 (2007)

8. H. R. Lissner, F. G. Evans. Experimental studies on the relation between acceleration and intracranial pressure changes in man. Surg Gynecol Obstet. 12, (1960)

9. J. Versace. A review of severity index. In: Proc of 15th Stapp Car Crash Conference. San Diego: Society of Automotive Engineers. (1971)

10. C. Gadd. Use of weighted-impulse criterion for estimating injury hazard. In: Proc of 10th Stapp Car Crash Conference. New Mexico: Society of Automotive Engineers. (1966)

11. J. Newman. A generalized acceleration model for brain injury threshold (GAMBIT). In: Proc of International IRCOBI Conference on the Biomechanics of Impact. Zurich: IRCOBI. (1986)

12. F. Bandak, R. A. Eppinger. A three-dimensional FE analysis of the human brain under combined rotational and translational accelerations. In: Proc of 38th Stapp Car Crash Conference. Florida: Society of Automotive Engineers. (1994)

13. J. Newman, N. Shewchenko, E. Welbourne. A proposed new biomechanical head injury assessment function the maximum power index. In: Proc of 44th Stapp Car Crash Conference. Atlanta: Society of Automotive Engineers. (2000)

14. A. Bartsch, E. Benzel, V. Miele. Hybrid III anthropomorphic test device (ATD) response to head impacts and potential implications for athletic headgear testing. Accid Anal Prev. 48, 5 (2012) 